\title{
Interactive comment on "Measurement report: Molecular composition and volatility of gaseous organic compounds in a boreal forest: from volatile organic compounds to highly oxygenated organic molecules" by Wei Huang et al.
}

Anonymous Referee \#1

Received and published: 2 February 2021

Comments on acp-2020-1257

In their manuscript "Measurement report: Molecular composition and volatility of gaseous organic compounds in a boreal forest: from volatile organic compounds to highly oxygenated organic molecules", the authors present data from summer in a boreal forest using a suite of state-of-the-art mass spectrometers. Overall, it is scientifically valid work, and advances the important work of trying to understand the different strengths and limitations of the many new CIMS approaches. My primary concern with 
of AMT. While the title pitches the science of the work, it is clear from the figures and most of the discussion that the bulk of this work is on intercomparisons between the 3 instruments(/instrument modes) and how they complement each other. While there are some plots of distributions and diurnals, etc., the focus of the discussion of these figures is again on the instrumentation. Overall, the bulk of the science here (and in my opinion, a lot of the highest value) is in the "Measurement Techniques" not the "Chemistry and Physics". In that context, I do have some technical concerns noted in my general comments below, but these are mostly addressable through changes in language and discussion and minor re-processing. I think this can and should be published with relatively minor revisions, but I'm not totally convinced that should happen in this specific journal.

\section{General comments}

(1) There are some minor english issues - nothing egregious but quite a few cases of odd sentence structures. One of the english-as-a-first-language authors on this work should copy edit more closely.

(2) In parameterizing Csat, Li et al. has a problem with nitrogen. The empirical approach was derived with very few nitrate groups, so treats nitrogen essentially as an amine. In environments where NO3 is expected to be a dominant form of organic nitrogen, this can bias the vapor pressure low by roughly two orders of magnitude per nitrogen atom. Probably not enough to change any conclusions, but with CHON representing roughly a quarter of the MION mass, it is probably enough to shift some distributions around a little. A recent paper in review in this journal describes this issue (https://acp.copernicus.org/preprints/acp-2020-1038/acp-2020-1038.pdf) , and proposes a solution by treating $\mathrm{NO} 3$ units a $\mathrm{OH}$ groups, following the approach used by Daumit et al. in their parameterization.

(3) While this paper focuses most heavily on intercomparisons between the instruments, considerations of some of the pitfalls of these tools are not discussed. For 
example, though the potential presence of isomers is discussed in a few places, it tends to be glossed over based on relatively weak assumptions (e.g., different isomers probably have different diurnals). While a few specific spots are described below, I would just more generally caution the authors to consider that it is quite likely that the presence of isomers is the rule, not the exception, at that different isomers may have significantly different instrument sensitivities, the the authors should keep this in mind as they interpret their results. Its not really clear to me why diurnals tend to be the metrics by which isomer composition is being compared - why not point-by-point correlations, which should be high if they are truly the same isomers? One suggestion is, while isoprene is lower than monoterpenes, you may see the C5-methyltetrols ( $\mathrm{C} 5 \mathrm{H} 12 \mathrm{O} 4)$. This specific species is helpful because there are not a lot of likely ways to draw that formula since it is saturated and a dominant isoprene product (though there are a few peroxide options), so if multiple intruments see it, it might give some benchmark as to how correlated ions might be when they are very likely the same set of isomers.

Similarly, the role of fragmentation in these results is not considered deeply, though PTR is known to fragment. What does this mean for measured mass? For instrument overlap? For average elemental composition? Is this related to the flat diurnal in Vocus $\mathrm{CHO}$ and $\mathrm{CHON}$ ? These instruments are amazing advances, but they do have limitations in their interpretation, and these limitations are not always deeply considered in this work.

Technical comments "VOC" is usually pluralized as VOCs when used in a plural sense. Line 70-71. This sentence isn't quite grammatically correct, re-word.

Line 73-76. Run-on sentence, somewhat confusing.

Line 74. Why isn't Interface capitalized in APi-ToF?

Discussion paper

Line 75. It's not clear to me: is MION just a switching reagent ionization approach, 
which has been shown previously using a PTR, but as applied to an API-ToF? Because it is discussed in the same "breath" as the the Vocus, my initial reading is that it is a functionality of the Vocus, but I gather that the MION instrument is a physically distinct ToF-CIMS. This confusion makes it a bit hard to understand or parse the rest of this paragraph. I think this paragraph just needs some editing and further clarification and detail.

Interactive

Line 108. "Finnish winter time" is confusing, since it is in spring. Perhaps "Finnish Winter Time" or "Eastern European Time (EET)" which I think is the general term for UTC+2 in Europe.

Line 126-129. Is the assumption the sulphuric acid represents the kinetic limit sensitivity, so is used as a floor for org? If so, that should be explicitly mentioned, otherwise it's not clear why the org sensitivity factors are being determined by sulphuric acid.

Line 134-137. Similar diurnal patterns is a poor approach to determining isomer content. Take, for example, monoterpenes, for which there are usually around a dozen isomers, but all are expected to have a similar diurnal. Point-to-point correlations (R2) might be a better metric than diurnals, but it will still suffer from this example issue (just perhaps less so). Since you are comparing across two different ionizations, this approach is perhaps a bit more reasonable (if an ionization scheme sees one group of isomers, the other one probably does too), but it is still has serious issues. Isomers can vary in their senstivity by an order of magnitude within an ionization scheme (e.g., iodide, Lee et al. CITE), so one ionization scheme could see one set of isomers with high sensitivity, and the other could see a different set with high sensitivity, but these could still have similar diurnals. All-in-all, I'm sympathetic to the need to do something about potential overlap and the uncertainties in bulk calibration of CIMS, but scaling one instrument to another based on diurnals is built on fairly shaky assumptions that need a more robust examination. Are there trends in correlations between ionizations as a funciton of ion elemental ratios that might allow you to tease out when they are seeing the same isomers and when they are not? Or any other features within the

Printer-friendly version

Discussion paper 
data? Simply put, similar diurnals is insufficient evidence for "likely to be the same species", and more caution is warranted in acting on this conclusion.

Line 155-157. This is a better/more conservative approach to handling overlap.

Line 182. A Pt100 should be defined/described

Interactive

Line 205. Is this the full list of compounds excluded, or just an example list? Is the full list provided somewhere? Would it be helpful to also add that data, for instance as Vocus_LL or some other signifier? That would be interested from an organic carbon budget perspective.

Line 233. Is this average composition of organic gases, or does it include ACSMmeasured organic particles?

Line 238. "followed-by groups" is not something l've seen before in written English

Lines 226-243. While PTR is fairly soft, it is known to have non-negligible fragmentation (Yuan et al., 2017, e.g., Figure 5 therein). How might this impact both the quantification of the total measurement by this instrument, and/or understanding of the elemental compositions? This issue of course does not involaidate the Vocus, or these measurements, but the effects of fragmentation and its impacts on the potential interpretation and conclusions in this work should be considered and discussed.

Line 268. It's not clear to me the C20 is necessarily diterpenes. While the SI does show some diterpenes (which is very exciting and interesting, and sadly buried in the SI), monoterpenes are known to dimerize and for C20 compounds. I note that the bar on C20 looks like it is mostly $\mathrm{O}>=12$, so highly oxygenated. Is this not just monoterpene dimers? It might provide some insight into the influence of monoterpene-dimers vs. diterpene-monomers to looks at distributions of oxygen number.

Printer-friendly version

Line 284-286. Why would this smearing occur for Vocus data, but not the other data? Is it related to the tendency for nitrates to fragment in PTR? 
Flgure 1. Why use ppb for inorganics and cm-3 for organics? Organic gases are more commonly reported as ppb.

Figures 6 and 7. I recognize why the authors chose to plot these distributions on a log scale, but a bar chart on a log scale is inherently inaccurate/confusing, especially a stacked bar chart. Because there is no "zero", drawing a line to zero on a bar chart creates a wholly arbitrary scaling, which means the bar size is no longer in any way proportional to quantity. Consider Figure 7 at $\log ($ Csat $)=7$. While roughly $99.9 \%$ of the concentrationis measured by the Vocus, more than half the bar is blue. At the same time, if the bars were stacked in the opposite order, MION-Br would be negligibly small sitting on top of the Vocus. Similarly, the scale on the $x$-axis could reasonably be altered to start at $10^{\wedge} 3$ or $10^{\wedge} 2$ instead of $10^{\wedge} 4$, and that choice would dramatically change the areas of only the bottom bar in the stack. What the solution here is, I'm not sure, but I strongly recommend the authors make some other style choice.

Is it worth splitting these figures across two figures? It seems to me that $6 \mathrm{a}$ and $7 \mathrm{a}$ are showing basically the same data - couldn't you should had 6b-d to Figure 7. Relatedly, though they seem to be plotting the same data, I can't reconcile them quantitatively. Again, as an example log(Csat) $=7$. In Figure $6 \mathrm{a}$, this looks like roughly: Vocus $15 \%$, $\mathrm{Br} 8 \%$, NO3 3\%. In Figure 7a, the ratio is Vocus: stack from $10^{\wedge} 7.5$ to $10^{\wedge} 10=10^{\wedge} 10$, $\mathrm{Br}$ : stack to $10^{\wedge} 7.5$, NO3:negligible. That is a ratio of $\mathrm{Vocus}: \mathrm{Br}=300: 1$ instead of 2:1. I wonder of this issue is related to the stacked log plot issue described above.

Interactive comment on Atmos. Chem. Phys. Discuss., https://doi.org/10.5194/acp-2020-1257, 2020. 